\title{
Evidence-based health solutions for challenges women face during pandemics like COVID-19: A systematic review protocol
}

Zhaohui Su ( $\sim$ szh@utexas.edu )

University of Texas Health Science Center at San Antonio https://orcid.org/0000-0003-2005-9504

Shelly Wagers

University of South Florida - Saint Petersburg

Emme Lopez

University of Texas Health Science Center at San Antonio

Lori Ann Terjesen

National Women's History Museum

Jaffar Abbas

Shanghai Jiao Tong University

Feng Shi

United Imaging Intelligence

Caifeng Wang

Shanghai Jiao Tong University School of Medicine

Yuyang Cai

Shanghai Jiao Tong University School of Medicine

\section{Protocol}

Keywords: COVID-19, coronavirus, pandemic, epidemic, intervention, women's health, mental health, domestic violence

Posted Date: September 1st, 2020

DOl: https://doi.org/10.21203/rs.3.rs-66220/v1

License: (c) (i) This work is licensed under a Creative Commons Attribution 4.0 International License.

Read Full License 


\section{Abstract \\ Background}

One of the most vulnerable populations to COVID-19 is women. Multiple factors associated with violence against women (i.e. sexual assault, domestic violence, homelessness) create an increased vulnerability for women during the COVID pandemic. Women also constitute the majority of older nursing home residents and healthcare workers (e.g., nurses), who have the most pronounced exposure to COVID-19. These factors combined with resource restraints like rationing and lack of access to healthcare can further exacerbate women's physical and psychological health issues. While literature has welldocumented challenges that women face during COVID-19, there is a lack of evidence-based solutions that have the potential to mitigate these difficulties. Therefore, to address this issue, we aim to conduct a systematic review of the literature to: (1) identify interventions designed for women in the context of pandemics, (2) describe the characteristics and effects of these interventions concerning the distinctive traits of women and pandemics, and (3) present evidence-based health solutions for women to mitigate challenges they face amid and beyond COVID-19.

\section{Methods}

A systematic review of literature will be conducted on databases including PubMed, PsycINFO on the EBSCO platform, CINAHL on the EBSCO platform, and Scopus, based on a search strategy developed in consultation with an experienced medical librarian. Titles, abstracts, and full-text articles will be screened against eligibility criteria developed a priori. The Preferred Reporting Items for Systematic Reviews and Meta-Analyses procedures will be adopted as the reporting framework, and data extracted (e.g., intervention details) will be evaluated by a multidisciplinary research team.

\section{Results}

NA for now-This is a protocol study.

\section{Conclusions}

Findings of this study will fill an important void in the literature. Considering that, in times of pandemic, women are especially subject to grim health disparities, like pronounced exposure to COVID-19, reproductive health issues, elevated domestic violence, increased mental health challenges, and lack of access to healthcare services, the need for evidence-based health solutions that could address these unique challenges is of paramount importance. A comprehensive understanding of the characteristics and effects of health solutions available to women in the context of pandemics can also help researchers 
identify areas of improvement regarding intervention design and development. This may further safeguard women's health and wellbeing amid pandemics like COVID-19 and beyond.

\title{
Study Protocol Registration:
}

\author{
PROSPERO CRD42020194003
}

\section{Background}

COVID-19 has uprooted healthcare as we know it [3]. To date, COVID-19 has claimed over 20.4 million confirmed cases and 745,000 deaths worldwide [4], leaving the world scrambling and struggling to find a solution [5-7]. Similar to the severe acute respiratory syndrome or SARS and Middle East respiratory syndrome or MERS, coronavirus disease 2019 or COVID-19 is a pandemic that has caused fear and uncertainty across continents [8-10]. Different from SARS and MERS, COVID-19 has greater transmissibility [11-13]. This, in turn, fuels its adverse impacts on societies at large as it leaves little time for governments across the world to develop an effective plan to tackle the pandemic. While SARS, MERS, and COVID-19 share similar virus characteristics [11-13], the epidemiological attributes of COVID19 make it more difficult to contain and control as the virus evolves [11-13], further allowing it to continue wreaking havoc across the globe.

Mounting evidence shows that women are among the hardest hit populations during the pandemic [1418], especially non-white racial or ethnic communities such as black and Hispanic women [19, 20]. A preliminary literature review indicates that factors influencing women's increased vulnerability to COVID19 can be grouped into five primary contributors: (1) pronounced exposure to COVID-19, (2) issues related to women's reproductive health, (3) elevated domestic violence, (4) increased mental health challenges, and (5) lack of access to healthcare services. A detailed illustration can be found in Fig. 1.

Related to the first, primary contributor studies have shown that older adults, especially older nursing home residents and healthcare workers (e.g., nurses), have the most pronounced exposure to COVID-19 $[1,2]$. Women make up the majority of both these populations $[1,21-23]$ leading them to an increased probability and disproportionate risk of contracting COVID-19. It is estimated that, worldwide, women make up $55 \%$ of the older adult ( $\geq 65$ years old) population, whereas over $61 \%$ of the 80 years and older population are women [24]. In the U.S., data show recent reports indicate that approximately $70.6 \%$ of nursing home residents are women [21]. Nursing home residents often live with frailty and underlying conditions [21], which makes them particularly susceptible to COVID-19 infection and deaths. Repeated research indicates that nursing homes are ground zero in COVID-19 [2]. As of July 30, 2020, 44\% of all COVID-19 deaths in the U.S. occurred in nursing homes, which translates into 62,925 lives lost in total [25]. Research also indicates that between $61.3-90.0 \%$ frontline healthcare professionals are women [1, $23,26]$ and, in addition to an elevated risk of direct exposure to confirmed cases of COVID-19, most of these healthcare professionals often face substantial mental health issues, such as anxiety, insomnia, and depression $[27,28]$. 
The other primary factors that contribute to women's pronounced vulnerability to COVID-19 are genderspecific [29-31] and are rooted in the historical and cultural social power imbalances that result from being a patriarchal society, which disproportionately affects women, such as lower incomes and job loss and violence against women [18,32,33]. Research indicates that women's employment is 1.8 times more likely to be in jeopardy due to COVID-19 compared to men. Even though women make up approximately $39 \%$ of the employed population globally, they count for $54 \%$ of all COVID-19-induced job losses [34]. Domestic violence victims are also disproportionately impacted by COVID-19. Violence against women, or domestic violence, could be understood as "all behaviors which are based on gender, hurt, and damage, resulted or possibly resulted in physical, sexual and mental damage and cause to oppress on women in social or private life and arbitrary restrict the freedom of women" [35]. Domestic violence constituted a health pandemic before the coronavirus outbreak [36-39]. Reports from the World Health Organization indicate that, globally, 1 in 3 women is or will become a victim of physical or sexual violence at some point in their relationship [40]. COVID-19 only serves to escalate this reality for women [32].

Mounting evidence shows that both incidence and death rates related to violence against women have soared during the COVID-19 outbreak [32, 41-45]. In Australia, even though there was a 40\% drop in overall crime rates during the outbreak, domestic violence calls have increased $5 \%$ [46]. Available evidence from European countries further indicates that there is a $60 \%$ spike of emergency calls from female domestic violence victims during the COVID-19 outbreak [33]. Disturbing reports from the United Kingdom (U.K.) suggest that between 23 March and 12 April, 2020, female deaths due to domestic violence almost doubled compared with average rates in the past decade [47]. Compounding this effect is that, citing COVID-19 infection risks, Governors in the U.S. have largely released perpetrators in jail for misdemeanors [48], a categorization under which most domestic cases fall. This, in turn, may further heighten the burden of fear and uncertainty some women shoulder amid COVID-19, if not increase their odds of physical harm as well.

Undoubtedly, both health issues related to reproductive health and elevated domestic violence women face can have a grim effect on women's health during COVID-19, especially their mental health conditions $[30,46,49-52]$. Repeated evidence indicates that women who are subject to domestic violence often suffer from traumatic brain injuries (TBI) [53-55], which could have a long-term effect on their mental health as well [56]. Examining the prevalence of TBI among a group of domestic violence victims, researchers found that $88 \%$ of the women studied experienced more than one injury, and $81 \%$ of them had experienced loss of consciousness due to their injuries [54]. Mounting research indicates that mental health challenges domestic violence victims face may be even more disheartening [57-59]. Results from a meta-analysis of 207 studies show that depression, anxiety, posttraumatic stress disorder, antisocial personality disorder, and borderline personality disorder are common among domestic violence victims [57]. However, due to spatial distancing measures and COVID-19 medical resources rationing policies, many traditional venues of help, such as face-to-face consultation, have either been delayed or cancelled $[46,50,60-62]$. In addition, it is well-documented that most domestic violence cases remain underreported or unreported, because a considerable number of women do not seek help $[32,46,50,62$, 63]. 
It is not clear whether and to what degree women may benefit from the increasing number of technologybased health solutions proposed during the time of COVID-19. Older adults, the majority of whom are women [24], often face issues associated with cognitive decline [64, 65], making them highly dependent on physically-based healthcare services [66-68]. Furthermore, many older women face concurrent issues, like visual impairment, hearing difficulties, or dual sensory loss $[69,70]$. The majority of existing technology-based health solutions fail to address these challenges given that they are primarily delivered or hosted on websites and mobile applications [71-73].

Situations may be even worse for older domestic abuse victims. Research suggests that, amongst those who have experienced concussions, a symptom common among domestic abuse victims, females are more likely to develop computer-screen intolerance due to brain-related photosensitivity issues. In other words, though technology-based solutions exist, due to lack of tailored design and consideration of older users [74-76], these solutions may have limited utility and functionality for older females. All of these barriers, in turn, can contribute to the most vulnerable of the population left with little to no access to services $[2,46,50,62]$, especially factoring in the digital divide experienced by people who face both health disparities and economic hardships which may further exacerbates these individuals' unmet healthcare needs [77-79]. These combined insights indicate that, while women face substantial health issues amid COVID-19, most of these issues may remain unsolved for a prolonged period of time due to lack of available health solutions $[46,50,62]$. This, undoubtedly will further worsen the dire situations women face on a daily basis $[46,50,62]$.

Overall, there is dearth of research that examines health solutions that have the potential to address health issues people face amid COVID-19 [80], particularly for women. Available evidence indicates that corollary interventions have the potential to address women's health issues. Take the case of interventions for human immunodeficiency virus (HIV). Researchers found that educator-led programs based on the Health Belief Model yielded significantly more positive behavioral outcomes for females living with HIV when compared to standard talk therapy [81]. One way to determine potentially effective interventions for women during COVID-19 is to systematically review literature in the context of infectious disease pandemics $[80,82,83]$ as they share attributes such as unpredictability (e.g., when, where, and how the crises unfold and fade) and destructiveness (e.g., uprooted lives and livelihoods) [11-13]. Communicable disease pandemics often share similar disaster preparedness procedures as well, like social distancing measures [84-87]. Thus, interventions designed for women in the context of pandemics like SARS and MERS may be able to shed light on solutions women can use amid COVID-19 as well.

To bridge this gap in literature, we aim to systematically review the literature to: (1) identify interventions designed for women in the context of pandemics, (2) describe the characteristics and effects of these interventions concerning the distinctive traits of women and pandemics, and (3) present evidence-based and practical health solutions for women to mitigate challenges they face amid and beyond COVID-19. Findings of this study will fill an important void in the literature. Considering that women are often subject to grim health disparities, the need for evidence-based health solutions that could address the unique challenges women face during COVID-19 is of paramount importance. A comprehensive understanding 
of the characteristics and effects of health solutions available to women in the context of pandemics can also help researchers identify areas of improvement regarding intervention design and development.

\section{Methods}

To safeguard research rigor, the review was registered with the International Prospective Register of Systematic Reviews database or PROSPERO (CRD42020194003) a priori. Furthermore, the Principles of the Preferred Reporting Items for Meta-Analysis protocol (PRISMA) was adopted to guide reporting of this systematic review [88]. As evidence indicates, these systematic review mechanisms are procedures needed to avoid potential study duplication [89, 90], enhance research rigor [91, 92], improve study comparability and replicability [93], and in turn, increase review quality and transparency [94].

\section{Eligibility criteria}

Eligibility criteria have two parts: inclusion and exclusion criteria. A list of inclusion criteria is presented in Table 1. In the context of this study, the term pandemic is used to refer to both epidemics and pandemics, which is defined as "the occurrence in a community or region of cases of an illness, specific health-related behavior, or health-related events clearly in excess of normal expectancy" [95]. Specifically, this study is interested in epidemics or pandemics that are related to infectious diseases, such as the Zika virus epidemic, the Ebola outbreak, the H1N1 epidemic, the SARS pandemic, the MERS epidemic, and the COVID-19 pandemic. The female gender and the term woman or women are used interchangeably in this study and is defined as individuals with the female biological attributes, including chromosomes, anatomy, and hormones [96]. An intervention is defined as a persuasive strategy or program that has the potential to induce positive changes in people's health-related attitudes and behavior. Overall, studies will be excluded if they: (1) did not focus on women or have $a \geq 50 \%$ female population (2) did not conduct in the context of an infectious disease-induced pandemic (3) did not empirically evaluate an intervention in the form of randomized controlled trials (e.g., technology-based or non-technology-based interventions), (4) did not report descriptions of the interventions studied), and (5) did not report empirical research findings (e.g., effects of the interventions).

\section{Search strategy}

Our key search terms centered on three concepts: women's health, interventions, and pandemics. The search term was developed with consultation with an academic librarian. An example PubMed search string is included in Table 2. Search strings will be subsequently applied to databases including PubMed, PsycINFO, CINAHL, and Scopus. The search will be conducted in September, 2020. In addition to database searches, we will also manually search reference lists of the included articles to identify additional eligible papers. 
An experienced medical librarian will search published literature and unpublished studies for records discussing technology-based health interventions, pandemics, and women. The librarian will create search strategies using a combination of key words and controlled vocabulary in: PubMed 1946-; CINAHL on the EBSCO platform 1937-; PsycINFO on the EBSCO platform 1927-; Scopus 1966-; and clinicaltrials.gov 1997- present. Search results will not be limited by language or year. No database limits or filters will be applied. An initial search string was built and tested in PubMed in August of 2020 and is included in Table 2.

\section{Study selection}

Upon search completion, citations will be uploaded to Rayyan [97], with duplicates removed. Titles and abstracts will be screened by two principle reviewers against the eligibility criteria. This process will be administered in a double-blind fashion, as Rayyan allows the reviewing process to be blinded. After removing ineligible articles, the same review process will be applied to full-text articles for further review. Detailed reasons for exclusion will be recorded in the PRISMA flowchart. Discrepancies between reviewers will be resolved via group discussions.

\section{Study quality assessment}

Quality of eligible studies will be evaluated with the Cochrane Collaboration evaluation framework to determine risk of bias [98]. In total, there are seven domains to the framework: (1) random sequence generation, (2) allocation concealment, (3) blinding of participants and personnel, (4) blinding of outcome assessment, (5) incomplete outcome data, (6) selective reporting, and (7) other source of bias. Only scores of the first five items will be used for the risk evaluation [98]. A study will only be considered having a low or a high risk of bias if a "low" rating was given on three or more of these five items $[98,99]$. Two reviewers will evaluate risk of bias of all included studies independently. Group discussions will be used to resolve any potential discrepancies till a consensus is reached.

\section{Data extraction and synthesis}

To address the research aim, a comprehensive list of data will be extracted, including study characteristics (e.g., country of origin, study methods, and research purpose), sample characteristics (e.g., age, race/ethnicity, and disease history), interventions evaluated (e.g., intervention stimuli, intervention exposure, and weather the design of the intervention material is tailored to women or the epidemic/pandemic context), outcome variables assessed (e.g., before-after health outcome changes), as well as principal research findings. Data extraction will be conducted by all authors. Descriptive analysis will be used to shed light on the patterns among included articles (e.g., country of origin distribution), whereas narrative synthesis will be adopted to investigate the characteristics and effects of the interventions. A preliminary search found great heterogeneity within studies. Therefore, meta-analysis will 
only be considered once a more grounded understanding of the characteristics of included articles is reached.

\section{Results}

NA for now: This is a protocol study

\section{Discussions}

Women belong to one of the most vulnerable communities to the COVID-19 pandemic [14-18]. While useful insights are available in the literature, there is a lack of evidence-based health solutions that can be applied to help women alleviate health issues they face amid COVID-19. Therefore, to address this issue, we aim to systematically review the literature to: (1) identify interventions designed for women in the context of pandemics, (2) describe the characteristics and effects of these interventions concerning the distinctive traits of women and pandemics, and (3) present evidence-based and practical health solutions for women to mitigate challenges they face amid and beyond COVID-19. Findings of our review will be able to offer timely solutions that have the potential to alleviate health challenges women shoulder amid the pandemic.

To our best knowledge, this is the first systematic review that examines evidence-based solutions developed and evaluated in the context of epidemics and pandemics, with the aim to find solutions that can shed light on issues women face amid COVID-19. We expect findings of this study can help women better shoulder the adverse impacts of the pandemic. Based on effective solutions identified from the planned review, results of this study can also help researchers better design and develop health solutions that are tailored to the unique challenges women face amid COVID-19. Furthermore, we also expect to find potential areas for improvement within the literature as directions for future research endeavors. Preliminary search results indicate that interventions tailored to women in the context of epidemics or pandemics are often not technology-based, which indicates that their applicability in the context of COVID-19 might be compromised. Technology-based interventions can be understood as "the use of technology to manage or support health promotion strategies that aim to produce accessible and affordable health solutions to a target audience" [100]. Considering the physical constraints introduced by measures such as lockdowns and social distancing, a lack of technology integration among available health solutions may limit their abilities to mitigate health challenges women face amid COVID-19. Overall, we believe findings of the planned review will be able to offer evidence needed to address health issues women face amid COVID-19 and beyond.

\section{Conclusions}

NA for now: This is a protocol study

\section{List Of Acronyms}


COVID-19: coronavirus disease 2019

MERS: Middle East respiratory syndrome or MERS-CoV-2

SARS: severe acute respiratory syndrome or SARS-CoV

U.K.: United Kingdom

\section{Declarations}

\section{- Ethics approval and consent to participate}

o Not applicable.

- Consent for publication

o Not applicable.

- Availability of data and materials

o No.

- Competing interests

o None.

\section{- Funding}

o None.

\section{- Authors' contributions}

ZS developed the research idea and drafted the manuscript, SW, EL, LAT, JA, FS, CW, and YC reviewed and revised the manuscript.

\section{- Acknowledgements}

o The authors wish to express their gratitude to the editors and reviewers for their constructive input.

\section{References}


1. Wang $X$, et al. Clinical characteristics of 80 hospitalized frontline medical workers infected with COVID-19 in Wuhan, China. J Hosp Infect. 2020;105(3):399-403.

2. Barnett ML, Grabowski DC. Nursing homes are ground zero for COVID-19 pandemic. JAMA Health Forum. 2020;1(3):e200369-9.

3. Fauci AS, Lane HC, Redfield RR. Covid-19 - Navigating the uncharted. The New England Journal of Medicine. 2020;382(13):1268-9.

4. John Hopkins University. The COVID-19 global map. 2020 [cited 2020 August 26th]; Available from: https://coronavirus.jhu.edu/map.html.

5. Corey L, et al. A strategic approach to COVID-19 vaccine R\&D. Science. 2020;368(6494):948.

6. Graham BS. Rapid COVID-19 vaccine development. Science. 2020;368(6494):945.

7. Lurie N, et al. Developing Covid-19 vaccines at pandemic speed. N Engl J Med. 2020;382(21):196973.

8. The L. COVID-19: learning from experience. Lancet. 2020;395(10229):1011.

9. Pfefferbaum B, North CS. Mental health and the Covid-19 pandemic. N Engl J Med. 2020;383(6):510-2.

10. Mertens G, et al. Fear of the coronavirus (COVID-19): Predictors in an online study conducted in March 2020. J Anxiety Disord. 2020;74:102258.

11. Petrosillo N, et al. COVID-19, SARS and MERS: Are they closely related? Clin Microbiol Infect. 2020;26(6):729-34.

12. Fani M, Teimoori A, Ghafari S. Comparison of the COVID-2019 (SARS-CoV-2) pathogenesis with SARS-CoV and MERS-CoV infections. Future Virology. 2020. 10.2217/fvl-2020-0050. p.

13. Petrosillo N, et al. COVID-19, SARS and MERS: are they closely related? Clin Microbiol Infect. 2020;26(6):729-34.

14. Wenham C, Smith J, Morgan R. COVID-19: The gendered impacts of the outbreak. The Lancet. 2020;395(10227):846-8.

15. Bali S, et al. Off the back burner: Diverse and gender-inclusive decision-making for COVID-19 response and recovery. BMJ Global Health. 2020;5(5):e002595.

16. McLaren HJ, et al., Covid-19 and women's triple burden: Vignettes from Sri Lanka, Malaysia, Vietnam and Australia. Social Sciences, 2020. 9(5).

17. John N, et al., Lessons Never Learned: Crisis and gender-based violence. Developing World Bioethics, 2020. $\mathrm{n} / \mathrm{a}(\mathrm{n} / \mathrm{a})$.

18. Mazza $\mathrm{M}$, et al. Danger in danger: Interpersonal violence during COVID-19 quarantine. Psychiatry Res. 2020;289:113046.

19. Webb Hooper M, Nápoles AM, Pérez-Stable EJ. COVID-19 and Racial/Ethnic Disparities. JAMA. 2020;323(24):2466-7.

20. Bowleg L. We're not all in this together: On COVID-19, intersectionality, and structural inequality. American journal of public health. 2020;110(7):917-7. 
21. U.S. Department of Health And Human Services, Long-term care providers and services users in the United States,. 2015-2016, in Vital and Health Statistics. 2019, Centers for Disease Control and Prevention.

22. Friedberg L, et al. Long-term care: How big a risk? Boston: Center for Retirement Research; 2014.

23. Mo Y, et al. Work stress among Chinese nurses to support Wuhan in fighting against COVID-19 epidemic. J Nurs Manag. 2020;28(5):1002-9.

24. United, Nations. World population prospects 2019 highlights. Editor: Department of Economic and Social Affairs; 2020. United Nations.

25. Kaiser Family Foundation, State data and policy actions to address coronavirus. 2020, Kaiser Family Foundation.

26. Ong JJY, et al. Headaches associated with personal protective equipment - A cross-sectional study among frontline healthcare workers during COVID-19. Headache. 2020;60(5):864-77.

27. Lai J, et al. Factors associated with mental health outcomes among health care workers exposed to coronavirus disease 2019. JAMA Network Open. 2020;3(3):e203976-6.

28. Cai Q, et al. The mental health of frontline and non-frontline medical workers during the coronavirus disease 2019 (COVID-19) outbreak in China: A case-control study. J Affect Disord. 2020;275:210-5.

29. Bayefsky MJ, Bartz D, Watson KL. Abortion during the Covid-19 Pandemic - Ensuring access to an essential health service. N Engl J Med. 2020;382(19):e47.

30. Hussein J. COVID-19: What implications for sexual and reproductive health and rights globally? Sexual Reproductive Health Matters. 2020;28(1):1746065.

31. Baud D, et al. Second-trimester miscarriage in a pregnant woman with SARS-CoV-2 infection. JAMA. 2020;323(21):2198-200.

32. Peterman A, et al., Pandemics and violence against women and children, in CGD Working Paper. 2020, Center for Global Development: Washington, DC.

33. Mahase E. Covid-19: EU states report $60 \%$ rise in emergency calls about domestic violence. BMJ. 2020;369:m1872.

34. McKinsey G, Institute, COVID-19 and gender equality: Countering the regressive effects. 2020, McKinsey Global Institute.

35. United Nations. Declaration on the elimination of violence against women in United Nations General Assembly, Editor. 1993.

36. Crenshaw K. Mapping the margins: Intersectionality, identity politics, and violence against women of color. Stan L Rev. 1990;43:1241.

37. Johnson MP. Patriarchal terrorism and common couple violence: Two forms of violence against women. Journal of Marriage Family. 1995;57(2):283-94.

38. Heise L. Violence against women: The hidden health burden. World health statistics quarterly. 1993;46(1):78-85. 
39. Heise L, Ellsberg M, Gottemoeller M. Ending violence against women, in Population Reports. 1999. p. 1.

40. World Health Organization. Global and regional estimates of violence against women: Prevalence and health effects of intimate partner violence and nonpartner sexual violence. 2013.

41. United Nations Development Programme. Gender-based violence and COVID-19. New York: United Nations; 2020.

42. Roesch $\mathrm{E}$, et al. Violence against women during covid-19 pandemic restrictions. BMJ. 2020;369:m1712.

43. Allen-Ebrahimian B. China's domestic violence epidemic. 2020; Available from: https://www.axios.com/china-domestic-violence-coronavirus-quarantine-7b00c3ba-35bc-4d16-afddb76ecfb28882.html.

44. Taub A. A new Covid-19 crisis: Domestic abuse rises worldwide, in The New York Times. New York: NY; 2020.

45. Wagers S. Domestic violence growing in wake of coronavirus outbreak. 2020 [cited 2020 August 18th]; Available from: https://theconversation.com/domestic-violence-growing-in-wake-ofcoronavirus-outbreak-135598.

46. Usher K, et al. Family violence and COVID-19: Increased vulnerability and reduced options for support. International Journal of Mental Health Nursing. 2020;29(4):549-52.

47. Grierson J, Domestic abuse killings 'more than double' amid covid-19 lockdown, in Guardian. 2020: London, UK.

48. Carrega C, Katersky A. Inmates charged with violent crimes poised for release due to COVID-19. 2020 [cited 2020 August 18th]; Available from: https://abcnews.go.com/US/alleged-violent-offenderspoised-release-rikers-island-due/story?id=69863436.

49. Hall KS, et al. Centring sexual and reproductive health and justice in the global COVID-19 response. The Lancet. 2020;395(10231):1175-7.

50. Bradbury-Jones C, Isham L. The pandemic paradox: The consequences of COVID-19 on domestic violence. J Clin Nurs. 2020;29(13-14):2047-9.

51. Galea S, Merchant RM, Lurie N. The mental health consequences of COVID-19 and physical distancing: The need for prevention and early intervention. JAMA Internal Medicine. 2020;180(6):817-8.

52. Xiang Y-T, et al. Timely mental health care for the 2019 novel coronavirus outbreak is urgently needed. The Lancet Psychiatry. 2020;7(3):228-9.

53. Valera EM, et al. White matter correlates of mild traumatic brain injuries in women subjected to intimate-partner violence: A preliminary study. J Neurotrauma. 2019;36(5):661-8.

54. Zieman G, Bridwell A, Cárdenas JF. Traumatic brain injury in domestic violence victims: A retrospective study at the barrow neurological institute. J Neurotrauma. 2017;34(4):876-80. 
55. St Ivany A, et al. Extreme control and instability: Insight into head injury from intimate partner violence. Journal of Forensic Nursing. 2018;14(4):198-205.

56. Ivany AS, et al. Living in fear and prioritizing safety: Exploring women's lives after traumatic brain injury from intimate partner violence. Qual Health Res. 2018;28(11):1708-18.

57. Spencer $C$, et al. Mental health factors and intimate partner violence perpetration and victimization: $A$ meta-analysis. Psychology of Violence. 2019;9(1):1-17.

58. Riedl D, et al. Domestic violence victims in a hospital setting: Prevalence, health impact and patients' preferences - Results from a cross-sectional study. European Journal of Psychotraumatology. 2019;10(1):1654063.

59. Karakurt G, et al. Mining electronic health records data: Domestic violence and adverse health effects. J Fam Violence. 2017;32(1):79-87.

60. Farrell TW, et al. Rationing limited healthcare resources in the COVID-19 era and beyond: Ethical considerations regarding older adults. J Am Geriatr Soc. 2020;68(6):1143-9.

61. Emanuel EJ, et al. Fair allocation of scarce medical resources in the time of Covid-19. N Engl J Med. 2020;382(21):2049-55.

62. van Gelder N, et al. COVID-19: Reducing the risk of infection might increase the risk of intimate partner violence. EClinicalMedicine. 2020;21:100348-8.

63. Gulati G, Kelly BD. Domestic violence against women and the COVID-19 pandemic: What is the role of psychiatry? Int J Law Psychiatry. 2020;71:101594.

64. Kaup AR, et al. Trajectories of depressive symptoms in older adults and risk of dementia. JAMA Psychiatry. 2016;73(5):525-31.

65. Amjad H, et al. Underdiagnosis of dementia: An observational study of patterns in diagnosis and awareness in US older adults. J Gen Intern Med. 2018;33(7):1131-8.

66. Wang H, et al. Dementia care during COVID-19. The Lancet. 2020;395(10231):1190-1.

67. Palese A, et al. Self-feeding dependence incidence and predictors among nursing home residents: Findings from a 5 year retrospective regional study. Nurs Health Sci. 2019;21(3):297-306.

68. Payne M, Morley JE. Dysphagia, dementia and frailty. The Journal of Nutrition Health Aging. 2018;22(5):562-5.

69. Chen SP, Bhattacharya J, Pershing S. Association of vision loss with cognition in older adults. JAMA Ophthalmology. 2017;135(9):963-70.

70. Chan T, et al. Estimates of incidence and prevalence of visual impairment, low vision, and blindness in the United States. JAMA Ophthalmol. 2018;136(1):12-9.

71. Volosyak I, Gembler F, Stawicki P. Age-related differences in SSVEP-based BCI performance. Neurocomputing. 2017;250:57-64.

72. Dobres J, et al. The effects of visual crowding, text size, and positional uncertainty on text legibility at a glance. Appl Ergon. 2018;70:240-6. 
73. McCloskey D. The importance of ease of use, usefulness, and trust to online consumers: An examination of the technology acceptance model with older customers. J Organ End User Comput. 2006;18:47-65.

74. Chun YJ, Patterson PE. A usability gap between older adults and younger adults on interface design of an Internet-based telemedicine system. Work. 2012;41(Suppl 1):349-52.

75. Stronge AJ, Rogers WA, Fisk AD. Human factors considerations in implementing telemedicine systems to accommodate older adults. Journal of Telemedicine Telecare. 2007;13(1):1-3.

76. Narasimha S, et al. Designing Telemedicine Systems for Geriatric Patients: A Review of the Usability Studies. Telemed J E Health. 2017;23(6):459-72.

77. Dixon LJ, et al. Gendered space: The digital divide between male and female users in Internet public access sites. Journal of Computer-Mediated Communication. 2014;19(4):991-1009.

78. Rebecca PY, et al., Mapping the two levels of digital divide: Internet access and social network site adoption among older adults in the USA. Information, Communication and Society, 2016. 19(10).

79. Smith B, Magnani JW. New technologies, new disparities: The intersection of electronic health and digital health literacy. Int J Cardiol. 2019;292:280-2.

80. Li W, et al. Public health education for parents during the outbreak of COVID-19: A rapid review. Annals of Translational Medicine. 2020;8(10):628.

81. Jozani ZB, et al. The effects of an educational program based on the health belief model on information-motivation-skill-behavioral skills among women living with human immunodeficiency virus. J Educ Health Promot. 2019;8:252.

82. McGlone MS, et al. Don't let the flu catch you: Agency assignment in printed educational materials about the H1N1 influenza virus. J Health Commun. 2013;18(6):740-56.

83. Rubinstein $\mathrm{H}$, et al. Public preferences for vaccination and antiviral medicines under different pandemic flu outbreak scenarios. BMC Public Health. 2015;15(1):190.

84. Peeri NC, et al., The SARS, MERS and novel coronavirus (COVID-19) epidemics, the newest and biggest global health threats: What lessons have we learned? International Journal of Epidemiology, 2020.

85. Chu DK, et al. Physical distancing, face masks, and eye protection to prevent person-to-person transmission of SARS-CoV-2 and COVID-19: A systematic review and meta-analysis. The Lancet. 2020;395(10242):1973-87.

86. Guarner J. Three emerging coronaviruses in two decades. Am J Clin Pathol. 2020;153(4):420-1.

87. Zhang L, Liu Y. Potential interventions for novel coronavirus in China: A systematic review. Journal of medical virology. 2020;92(5):479-90.

88. Moher D, et al. Preferred Reporting Items for Systematic Reviews and Meta-Analyses: The PRISMA statement. PLoS Medicine. 2009;6(7):e1000097.

89. Stewart L, Moher D, Shekelle P. Why prospective registration of systematic reviews makes sense. Syst Rev. 2012;1:7. 
90. Chang SM, Slutsky J. Debunking myths of protocol registration. Syst Rev. 2012;1:4.

91. Xu C, et al. Protocol registration or development may benefit the design, conduct and reporting of dose-response meta-analysis: Empirical evidence from a literature survey. BMC Med Res Methodol. 2019;19(1):78.

92. dos Santos MBF, et al. Protocol registration improves reporting quality of systematic reviews in dentistry. BMC Med Res Methodol. 2020;20(1):57.

93. Shokraneh F. Reproducibility and replicability of systematic reviews. World Journal of Meta-Analysis, 2019. 7(3).

94. Ramstrand $\mathrm{N}$, et al. Promoting quality and transparency in clinical research. Prosthet Orthot Int. 2019;43(5):474-7.

95. Porta M. A dictionary of epidemiology. 6th ed. Oxford: Oxford University Press; 2014.

96. Census Bureau US. Gender. Glossary 2020 [cited 2020 August 1st]; Available from: https://www.census.gov/glossary/\#term_Gender.

97. Ouzzani M, et al., Rayyan - A web and mobile app for systematic reviews. Systematic Reviews, 2016. 5(210).

98. Higgins JP, et al., Cochrane handbook for systematic reviews of interventions. 2019: John Wiley \& Sons.

99. van Tulder $\mathrm{M}$, et al. Updated method guidelines for systematic reviews in the cochrane collaboration back review group. Spine (Phila Pa 1976). 2003;28(12):1290-9.

100. Su Z, et al., Understanding technology-based interventions for caregivers of cancer patients: $A$ systematic review-based concept analysis. Journal of Medical Internet Research, 2020.

\section{Tables}

Table 1. Study inclusion criteria 


\section{Category Criteria}

Study Women who are 18 years and older ( $\geq 50 \%$ of the whole population studied) population

Study Infectious disease-induced pandemics

context

Health Health solutions are defined as non-pharmaceutical interventions that are designed to solution change women's disaster preparedness amid pandemics

Key Detailed descriptions of the health solutions (i.e., purpose of the intervention, variable intervention stimuli, application of the interventions, intervention exposure, outcome variables assessed/measured, and weather the design of the intervention material is tailored to women or the epidemic/pandemic context)

Study type Original research (i.e., research that reports original and empirical research findings)

Research Randomized controlled trials design

Outcome Empirical reporting of the characteristics and effect of the health solutions (i.e., beforeafter effects on women's mental health)

Table 2. Example PubMed search string 


\begin{tabular}{|ll|}
\hline Concept & Search string \\
Women & \\
& "women"[MeSH] OR "women"[TIAB] OR "woman"[TIAB] OR "women s" [TIAB] OR \\
& "woman s"[TIAB] OR "female"[MeSH] OR "female"[TIAB] OR "females"[TIAB] OR \\
& "Mothers"[Mesh] OR "Surrogate Mothers"[Mesh] OR motherhood[TIAB] OR \\
& mothering[TIAB] OR mother[TIAB]
\end{tabular}

\section{Figures}




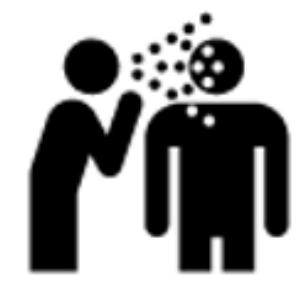

Pronounced Exposure to COVID-19

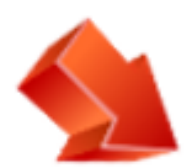

Issues Related to Women's

Women amid COVID-19

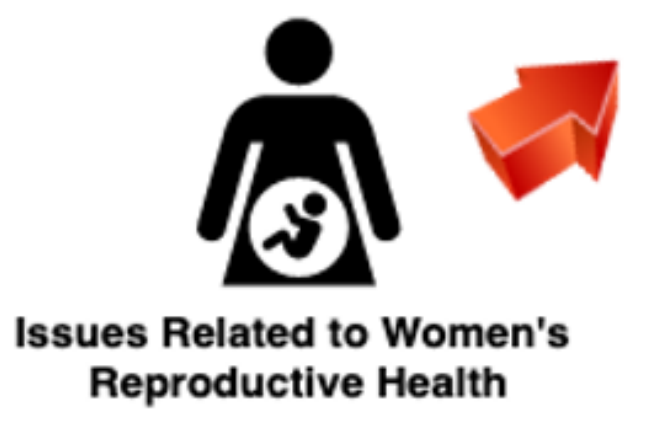

Reproductive Health
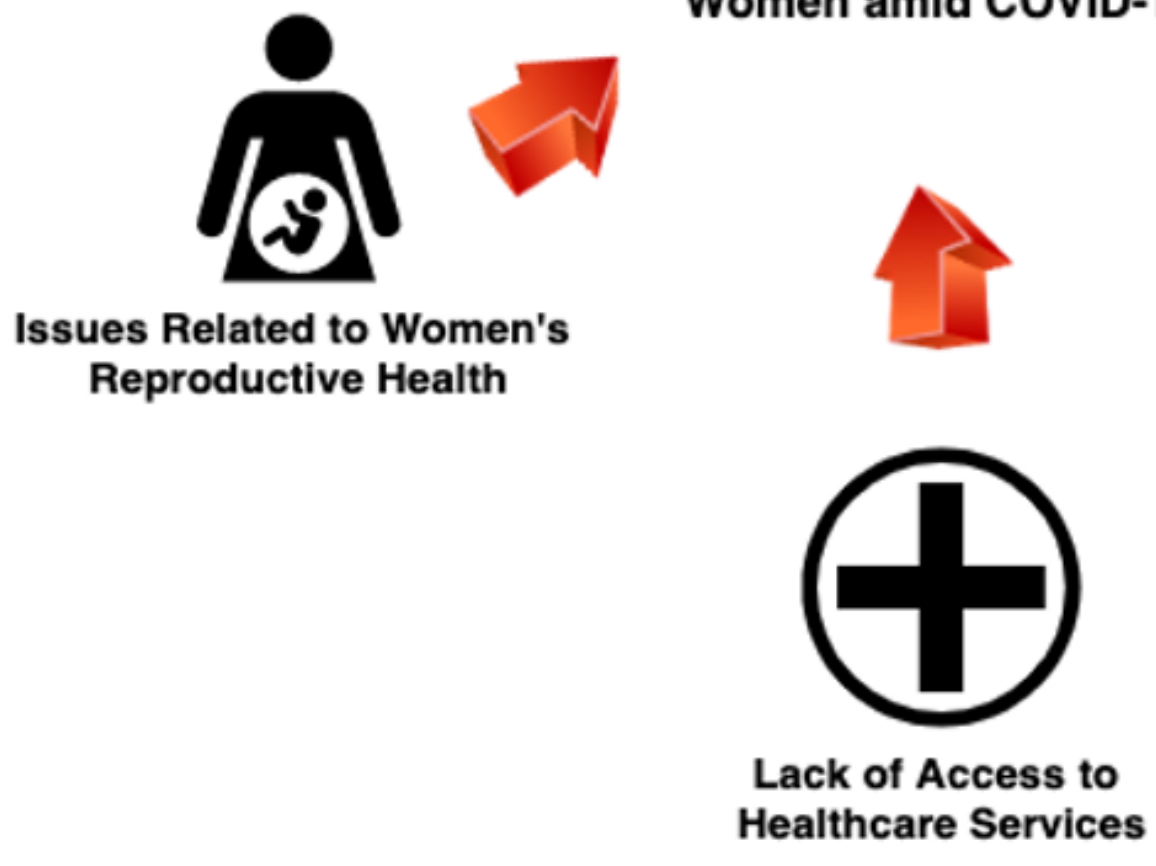

Lack of Access to Healthcare Services
Increased Mental Health Challenges

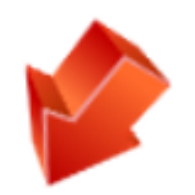

Figure 1

Main contributors to women's vulnerability to COVID-19

\section{Supplementary Files}

This is a list of supplementary files associated with this preprint. Click to download.

- PandemicSolutionsPRISMA2009checklist.doc 